\title{
TOLERANCIA Y COMPLICACIONES DE LA BIOPSIA TRANSRECTAL ECODIRIGIDA PROSTÁTICA AMPLIADA A DIEZ CILINDROS. PAPEL DEL BLOQUEO DE HACES NEUROVASCULARES CON LIDOCAÍNA.
}

\author{
Rafael Rodríguez-Patrón Rodríguez, Teodoro Mayayo Dehesa, Francisco Javier Burgos Revilla?, \\ Mónica Alonso González², Ricardo García González y Alberto Lennie Zucharino.
}

Unidad de Ecografía. 'Universidad de Alcalá de Henares. Madrid. ${ }^{2}$ Técnico de salud Área 8. Madrid. España.

\begin{abstract}
Resumen.- OBJETIVO: El creciente interés por ampliar el número de muestras obtenidas durante la biopsia transrectal ecodirigida prostática nos ha hecho evaluar la tolerancia y complicaciones de la biopsia ampliada con y sin bloqueo de los haces neurovasculares de esta modalidad diagnóstica.
\end{abstract}

MÉTODO: Un grupo de 222 pacientes se sometieron a biopsia prostática con intención de obtener 10 cilindros de tejido. Tras la evaluación de los primeros 50 pacientes realizados sin anestesia se decidió continuar practicando bloqueo de los haces neurovasculares con lidocaina al $2 \%$, evaluando comparativamente los resultados de tolerancia en ambos grupos y la incidencia de complicaciones y efectos secundarios en su conjunto.

RESULTADOS: No se pudo completar el número de muestras previsto en el $16 \%$ de pacientes sin anestesia frente al 2,33\% con anestesia $(p<0,002)$, la prueba resultó dolorosa o muy dolorosa en el 10,9 y 1,9\% respectivamente $(p<0,0002)$. La puntuación en la escala

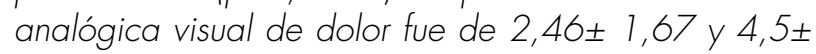
2,11 según se hubiera usado anestésico o no con reacciones vagales leves-moderadas en el $28 \%$ de estos últimos frente al 7,7\% si se administró lidocaina. La rectorragia fue la complicación más preocupante siendo moderada-importante en el 4,7\% precisando de ingreso en un $2 \%$ de pacientes.

CONCLUSIONES: El incremento del número de muestras en la biopsia transrectal ecodirigida se puede asociar a una mayor frecuencia de complicaciones sobre todo hemorrágicas y precisa de la aplicación de anestesia local por su peor tolerancia, si bien el bloqueo de haces neurovasculares con lidocaina es muy efectivo parra disminuir el dolor asociado a las punciones.

Palabras clave: Biopsia Ampliada. Bloqueo de haces neurovasculares. Complicaciones. Tolerancia.

Summary.- OBJECTIVES: The growing interest on increasing the number of biopsy samples during ultrasound guided prostatic biopsies moved us to evaluate the tolerability and complications of the extensive biopsy with/without blockage of neurovascular bundles.

METHODS: A group of 222 patients underwent prostatic biopsy with the aim to obtain 10 cores on each. After evaluation of the first 50 cases performed without anesthesia, decision was taken to proceed with neurovascular blockage with 2\% lidocaine, comparatively evaluating both groups for results on tolerability, complications and global adverse events.

RESULTS: The aimed number of cores could not be completed in $16 \%$ of the patients without anesthesia in 
comparison with $2.33 \%$ with anesthesia $(p<0.002)$; the biopsy was qualified as painful or very painful by $10.9 \%$ and $1.9 \%$ respectively $(p<0.0002)$. The results of the visual analogical scale for pain were $2.46 \pm 1.67$ and $4.5 \pm 2.11$ for the anesthesia / without anesthesia groups respectively, with mild-moderate vagal reactions in $28 \%$ of these latter in comparison with $7.7 \%$ in patients receiving lidocaine. Rectal bleeding was the most worrying complication, being moderate-severe in $4.7 \%$ of the patients, with $2 \%$ hospital admission.

CONCLUSIONS: The increase in the number of ultrasound guided prostatic biopsy samples may be associated with a higher frequency of complications, mainly bleeding, and requires the application of local anesthesia due to worse tolerance. Neurovascular bundle blockage with lidocaine is very effective to diminish biopsy associated pain.

Keywords: Extended biopsy. Neurovascular bundle blockage. Complications. Tolerability.

\section{INTRODUCCIÓN}

La Biopsia Prostática se realiza desde hace décadas. Aunque la vía transrectal se describió inicialmente a finales de los años 30 , no ganó demasiados adeptos hasta finales de los 60 cuando volvió a mostrarse interés por la Punción Aspiración con Aguja Fina (PAAF) que disminuía de forma importante la posibilidad de complicaciones infecciosas. Durante las décadas de los 60 y 80 se prefería la PAAF a cualquier técnica de biopsia dado que esta necesitaban en muchas ocasiones anestesia y tenían un porcentaje de complicaciones en torno al $10 \%(1,2)$. Con la introducción de la Ecografía Transrectal, los avances en profilaxis antibiótica y sobre todo la aparición de los dispositivos de disparo automático precargadas con agujas tipo Tru-Cut, que mejoraban la tolerancia al procedimiento, la Biopsia Transrectal Ecodirigida Prostática (BTE) se transformó en la referencia para el diagnóstico del Cáncer Prostático (CP). En cualquier caso a pesar de la mejoría de la técnica no se encuentra libre de complicaciones sobre todo hemorrágicas e infecciosas (3).

Por otro lado, en los últimos años existe un interés creciente en la realización de biopsias con mayor número de muestras en un intento de incrementar la capacidad diagnóstica de la prueba sin que hasta el momento quede claro el número idóneo de cilindros. Dada la buena experiencia que disponía nuestra Unidad con la Biopsia Sextante en cuanto a tolerancia y complicaciones se pretendían evaluar estos aspectos en un grupo de pacientes sometidos a Biopsia Ampliada (BA) a 10 cilindros de tejido.

\section{MATERIAL Y MÉTODO}

El grupo de estudio está formado por 222 pacientes que se sometieron a BA con 10 cilindros prostáticos. Inicialmente no se planteó el empleo de anestesia local dada la buena tolerancia que en nuestra experiencia presentaba la biopsia sextante, pero después de 50 casos al observar los resultados preliminares de tolerancia y porcentaje de pacientes en los que no pudimos completar la toma de muestras se decidió continuar el estudio añadiendo anestesia local al procedimiento. A partir de aquí se denominará al grupo realizado sin anestesia como "GRUPO SA" y al otro "GRUPO CA". Para la anestesia se administró lidocaina al $2 \%$ sin vasoconstrictor, aplicando 5 $\mathrm{ml}$ en el ángulo que en cortes longitudinales forman la vesícula seminal y la base de la próstata a cada lado, a nivel de los haces neurovasculares. La introducción mediante una aguja 22 Gauge de $15 \mathrm{~cm}$ de longitud se controló ecográficamente.

Los pacientes recibían una preparación previa mediante la administración dos horas antes del procedimiento de un enema comercial de $250 \mathrm{cc}$, un comprimido de metamizol magnésico y $500 \mathrm{mg}$ de levofloxacino o ciprofloxacino por vía oral que sería continuado posteriormente cada $24 \circ 12$ horas respectivamente hasta completar 5 días de tratamiento. Esta preparación es análoga a la que se viene realizando desde el inicio de las biopsias transrectales por sextantes en nuestra unidad.

Para el estudio de la tolerancia y recogida de complicaciones y efectos secundarios se entregó a los pacientes un cuestionario que podían completar de forma inmediata o diferida, entregándolo en este caso al recoger el resultado de la anatomía patológica o por correo. Dicho cuestionario incluye una serie de preguntas sobre distintos aspectos de la prueba y una escala analógica visual de dolor.

El medico realizador de la prueba completó un cuestionario que incluía datos generales (edad, fármacos antiagregantes o anticoagulantes, presencia de patología o alteraciones anales, tono esfinteriano...) una evaluación subjetiva de la ansiedad del paciente antes de iniciar la prueba y de su tolerancia durante las distintas fases de la misma para poder establecer una comparación con la apreciación referida por el paciente. Finalizada la BTE se recogía también el sangrado rectal inmediato y la aparición de reacciones vagales $u$ otros efectos secundarios. 
Se contactó telefónicamente con los pacientes para evaluar las complicaciones y efectos secundarios entre 7 y 10 días después de la biopsia. Cuando no fue posible se intentó hasta más de un mes después de la prueba y hasta en 8 o 10 ocasiones en diferentes horarios. En cualquier caso, en el momento del alta se les facilitó un teléfono de contacto 24 horas para que comunicasen cualquier evento adverso. Fueron contactados 194 pacientes de los 222 del grupo. Se asume que los pacientes no localizados no debieron tener complicaciones de importancia puesto que ninguno se puso en contacto con nosotros pero se han excluido al estudiar las frecuencias.

Los cuestionarios de recogida de datos pueden verse en los anexos I-III.

Se realiza un estudio descriptivo y comparativo de la tolerancia de la biopsia con 10 cilindros entre el grupo realizado con anestesia y el grupo sin anestesia.

\section{RESULTADOS}

Los datos de tolerancia de ambos subgrupos recogidos por el realizador de la prueba se recogen en la Tabla I. No se encontraron diferencias significativas en nuestra apreciación subjetiva respecto a la ansiedad previa ni en la tolerancia al TR y paso del transductor pero sí en la apreciación de la tolerancia a la biopsia que fue significativamente mejor en los pacientes en los que se administró anestesia local $(p<0,0001)$.

En el "grupo SA" no se pudo completar la toma de 10 muestras en 8 pacientes $(16 \%)$, cuatro de ellos admitieron 8 muestras $(4+4)$, en 3 pacientes se obtuvieron 6 cilindros $(3+3)$ y en uno sólo $5(3+2)$. En el "grupo CA" ocurrió en 4 pacientes $(2,33 \%)$, uno de ellos no toleró siquiera el paso del transductor por lo que no se obtuvo ninguna muestra. En los tres restantes se obtuvieron 2, 4 y 6 tomas. La diferencia de pacientes en los que no se tomaron el número previsto de muestras entre ambos grupos fue significativa $(p<0,0002)$.

\section{ANEXO 1. CUESTIONARIO DE RECOGIDA DE DATOS GENERALES Y TOLERANCIA SEGÚN REALIZADOR.}

Nombre paciente:

№ biopsia: antiagregantes $\mathrm{SI} N \mathrm{NO}$ teléfono:

anticoagulantes: $\mathrm{SI} \mathrm{NO}$

\section{EXPLORACIÓN FÍSICA:}

$\begin{array}{llllll}\text { Hemorroides externas } & \text { SI } & \text { NO } & \text { Hemorroides internas } & \text { SI } & \text { NO } \\ \text { Fisura anal } & \text { SI } & \text { NO } & \text { Cirugía anal } & \text { SI } & \text { NO } \\ \text { Tono esfinteriano: normal alto } & \text { bajo } & \text { Estenosis canal } & \text { SI } & \text { NO }\end{array}$

Stress pre-test:

Tolerancia paso transductor:

Tolerancia biopsia ler lóbulo:

Tolerancia biopsia $2^{\circ}$ lóbulo:

Tolerancia global:

Tamaño prostático:

Se completó el número previsto?:

OBSERVACIONES INMEDIATAS:

HEMORRAGIA

1 (nada o leve)

1 (nada molesto)

1 (nada molesto)

1 (nada molesto)

1 (nada molesto)

1.Transductor limpio
2 (moderado) 3 (importante)

2 (molesto)

2 (molesto)

2 (molesto)

2 (molesto) 3(doloroso)

3(doloroso)

3 (doloroso)

3(doloroso)
4 (muy importante)

4 (muy doloroso)

4 (muy doloroso)

4 (muy doloroso)

4 (muy doloroso)

№ cilindros: dcho: izqdo:

SI NO

2. Transductor con sangre

3. Salida de sangre espontánea por ano

REACCIÓN VAGAL:

4. Pérdida espontánea abundante

1. Ninguna

2. Cuadro vegetativo leve (Palidez y sudoración)

3. C. Vegetativo importante (mareo prolongado, sudoración)

4. C. Vegetativo severo (pérdida conocimiento, convulsión) 


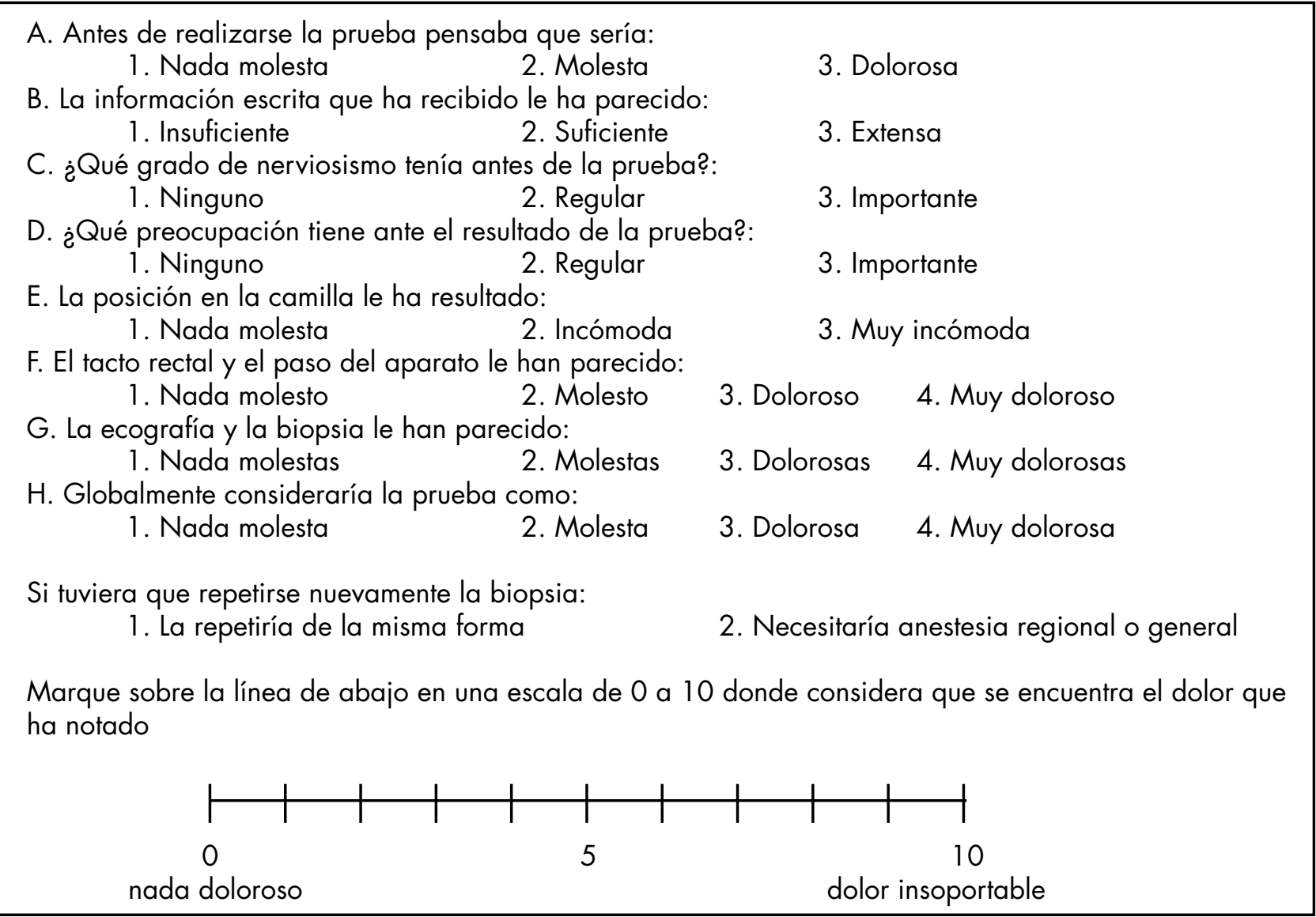

Del total de pacientes 206 (92,8\%) devolvieron el cuestionario de tolerancia aunque no todos contestaron a todos los apartados.

Al comparar las respuestas entre los grupos realizados $\sin$ y con anestesia no se encontraron diferencias significativas en las preguntas $A, B, C$ y $D$ (Anexo 2).

Existe sin embargo diferencia significativa en la apreciación de la posición en la camilla $(p<0,02)$. El TR y el paso del transductor resultó doloroso o muy doloroso en el $2,58 \%(n=4)$ de los pacientes del "grupo $\mathrm{CA}$ " frente al $10,87 \%(\mathrm{n}=5)$ en el "grupo $\mathrm{SA}^{\prime}$ $(p<0,009)$.

Consideraron la ecografía y la biopsia como dolorosa un $22,73 \%(n=10)$ del grupo SA frente a sólo el 4,52\%(n=7) del "grupo CA" ( $<<0,0001)$. En la apreciación global de la prueba vuelven a evidenciarse diferencias considerando la prueba dolorosa o muy dolorosa en un 10,87 vs $1,91 \%(p<0,0002)$.

Aunque una mayor proporción de pacientes del "grupo SA" considerarían necesaria algún tipo de anestesia ( $5 \%$ vs $0,69 \%)$, no se alcanzan diferencias significativas.

La media de puntuación en la Escala Visual Analógica de dolor para el "grupo CA" fue de $2,46 \pm 1,67$ mientras que en los realizados sin anestesia fue de 4,5 2,11 ( $p<0,0001)$.

Las Figuras 2 A-G muestran de forma visual la relación entre las respuestas a los distintos epígrafes en ambos grupos de pacientes.

No se apreciaron diferencias en el grado de sangrado rectal inmediato pero sí en las reacciones vagales que fueron más frecuentes en el grupo SA, presentando una reacción leve o moderada el $28 \%$ frente al $7,7 \%$ del grupo CA ( $p<0,0008)$.

Durante la administración de la anestesia local 6 pacientes $(3,48 \%)$ refirieron grados variables de mareo leve, alteraciones auditivas y sabor metálico en la boca o parestesias linguales. En todos los casos fue transitorio, desapareciendo antes de terminar la biopsia y no necesitó tratamiento específico. 
ANEXO 3. PROTOCOLO DE RECOGIDA DE COMPLICACIONES Y EFECTOS ADVERSOS.

COMPLICACIONES INFECCIOSAS

FIEBRE: 0 (no fiebre) 1 (fiebre $<38^{\circ}$ ) 2 (fiebre $>38^{\circ}$ )

MOMENTO DE APARICIÓN: DURACIÓN: días

TRATAMIENTO: 1. Cambio de antibiótico

2. Ingreso. Antibiótico I.V.

3. Otros

SI FIEBRE, ¿̇HA HECHO PROFILAXIS CORRECTAMENTE?:SI NO

COMPLICACIONES :

COMPLICACIONES POR SANGRADO:
RECTORRAGIA:
0 . Nada
1. Leve
2. rectorragia con coágulos/Paciente acude a urgencias y alta
3. Paciente requiere ingreso

TRATAMIENTO: 1. Observación

2.coagulación por rectoscopia
3. Cirugía
4. Transfusión
5. Taponamiento

HEMATURIA:

0. Nada

1. Leve

2. Aparición de coágulos

3. Retención urinaria por coágulos

OTRAS COMPLICACIONES

DIFICULTAD MICCIONAL: $\quad$ 0. Ninguna 1. Leve $\quad$ 2. Moderada

DIFICULTAD EVACUACIÓN: $\quad$ 0. Ninguna 1. Leve

$\begin{array}{lll} & \text { 2. Moderada } & \text { 3. Impo } \\ \text { ESCOZOR MICCIONAL: } & \text { 0. Ninguno } & \text { 1. Leve }\end{array}$

DOLOR ABDOMINAL: $\quad$ 0. Ninguno 1. Leve

2. Moderado 3. Importante

HEMOSPERMIA: SI NO NS/NC

OTROS:

TABLA I, DATOS SUBJETIVOS RECOGIDOS POR EL REALIZADOR SOBRE TOLERANCIA.

\begin{tabular}{|c|c|c|c|c|c|c|}
\hline & $\begin{array}{ll} & \text { GRADO } \\
\text { GRUPO } & \end{array}$ & 1 & 2 & 3 & 4 & $p$ \\
\hline \multirow{2}{*}{$\begin{array}{l}\text { ANSIEDAD } \\
\text { PRE-BTE }\end{array}$} & $\begin{array}{c}\text { SA } \\
n=50\end{array}$ & $\begin{array}{c}25 \\
(50,0 \%)\end{array}$ & $\begin{array}{c}16 \\
(32,0 \%)\end{array}$ & $\begin{array}{c}8 \\
(16,0 \%)\end{array}$ & $\begin{array}{c}1 \\
(2,0 \%)\end{array}$ & \\
\hline & $\begin{array}{c}C A \\
n=167\end{array}$ & $\begin{array}{c}65 \\
(38,9 \%)\end{array}$ & $\begin{array}{c}58 \\
(34,73 \%)\end{array}$ & $\begin{array}{c}41 \\
(24,55 \%)\end{array}$ & $\begin{array}{c}3 \\
(1,80 \%)\end{array}$ & n.s. \\
\hline \multirow{2}{*}{$\begin{array}{l}\text { TOLERANCIA AL } \\
\text { TACTO RECTAL } \\
\text { Y PASO DEL } \\
\text { TRANSDUCTOR }\end{array}$} & $\begin{array}{c}\mathrm{SA} \\
\mathrm{n}=50 \\
\end{array}$ & $\begin{array}{c}30 \\
(60,0 \%) \\
\end{array}$ & $\begin{array}{c}17 \\
(34,0 \%) \\
\end{array}$ & $\begin{array}{c}3 \\
(6,0 \%) \\
\end{array}$ & $\begin{array}{l}0 \\
2\end{array}$ & \\
\hline & $\begin{array}{c}C A \\
n=168 \\
\end{array}$ & $\begin{array}{c}89 \\
(52,98 \%) \\
\end{array}$ & $\begin{array}{c}59 \\
(35,12 \%) \\
\end{array}$ & $\begin{array}{c}18 \\
(10,7 \%) \\
\end{array}$ & $\begin{array}{c}(1,19 \%) \\
3 \\
\end{array}$ & n.s. \\
\hline \multirow{2}{*}{$\begin{array}{l}\text { TOLERANCIA A } \\
\text { LA BIOPSIA }\end{array}$} & $\begin{array}{c}\text { SA } \\
n=168 \\
\end{array}$ & $\begin{array}{c}12 \\
(24,0 \%) \\
\end{array}$ & $\begin{array}{c}18 \\
(36 \%) \\
\end{array}$ & $\begin{array}{c}17 \\
(34 \%) \\
\end{array}$ & $\begin{array}{c}(6 \%) \\
3 \\
\end{array}$ & \\
\hline & $\begin{array}{c}C A \\
n=50\end{array}$ & $\begin{array}{c}132 \\
(78,57 \%)\end{array}$ & $\begin{array}{c}25 \\
(14,88 \%)\end{array}$ & $\begin{array}{c}8 \\
(4,76 \%)\end{array}$ & $(1,79 \%)$ & $p<0,0001$ \\
\hline
\end{tabular}

$S A=\sin$ anestesia, $C A=$ con anestesia local 

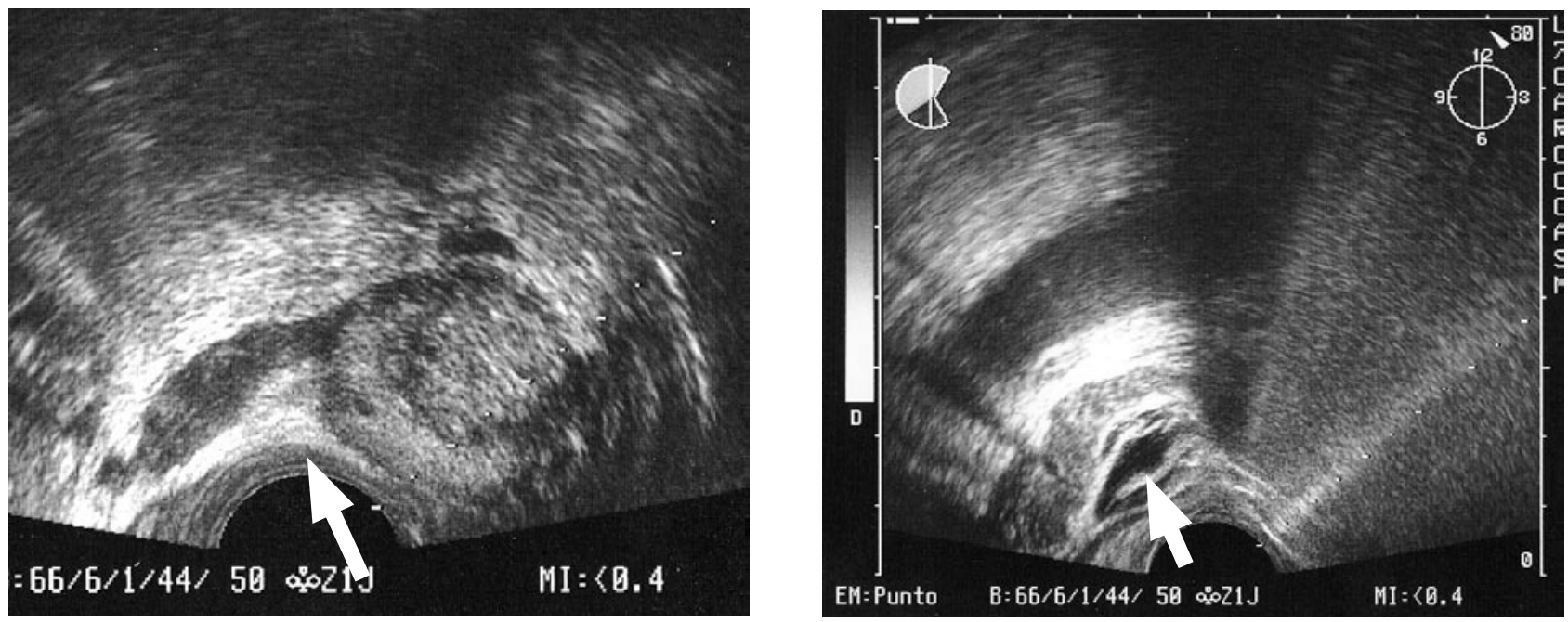

FIGURA 1. A) lugar de administración de anestésico local en el ángulo que forma la vesícula seminal y la base prostática (flecha). B) Habón de fármaco en el lugar indicado

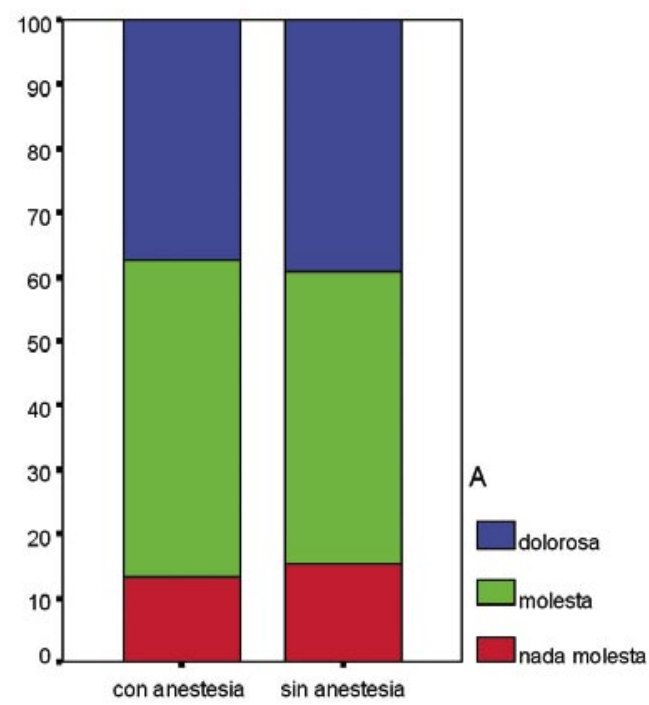

FIGURA 2A. Antes de realizar la prueba pensaba que sería

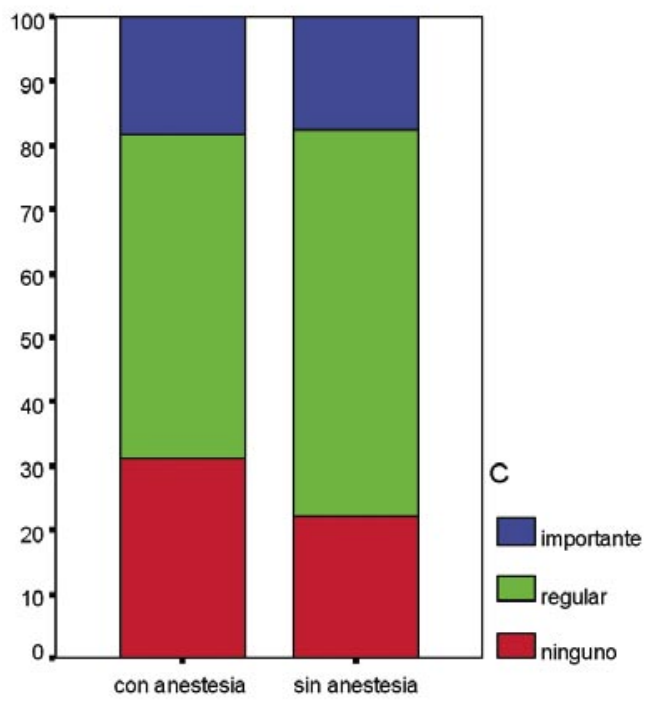

FIGURA 2C. ¿qué grado de ansiedad tenía antes de la prueba?

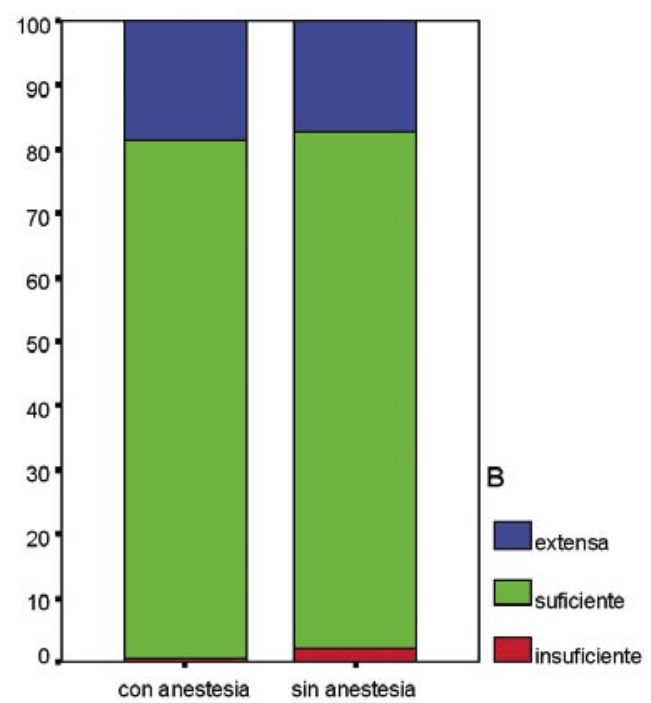

FIGURA 2B. La información que ha recibido le ha parecido

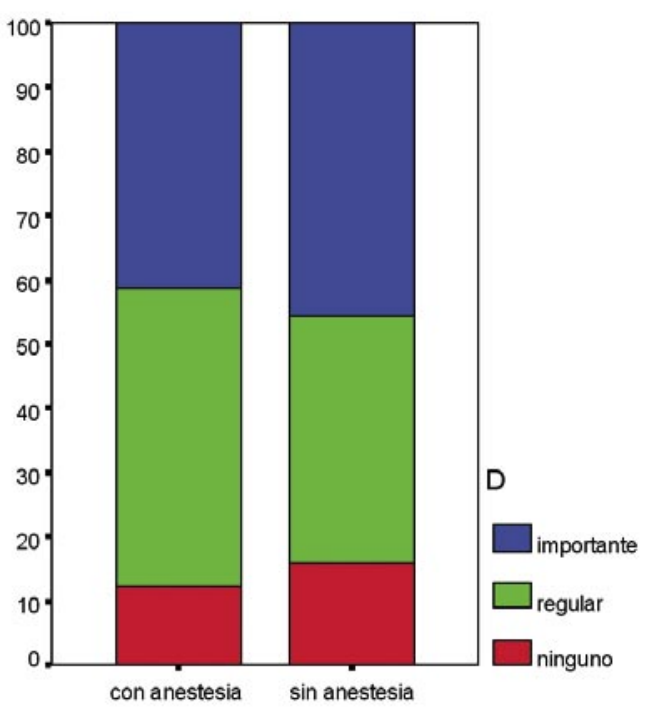

FIGURA 2D. La preocupación ante el resultado de la prueba es 


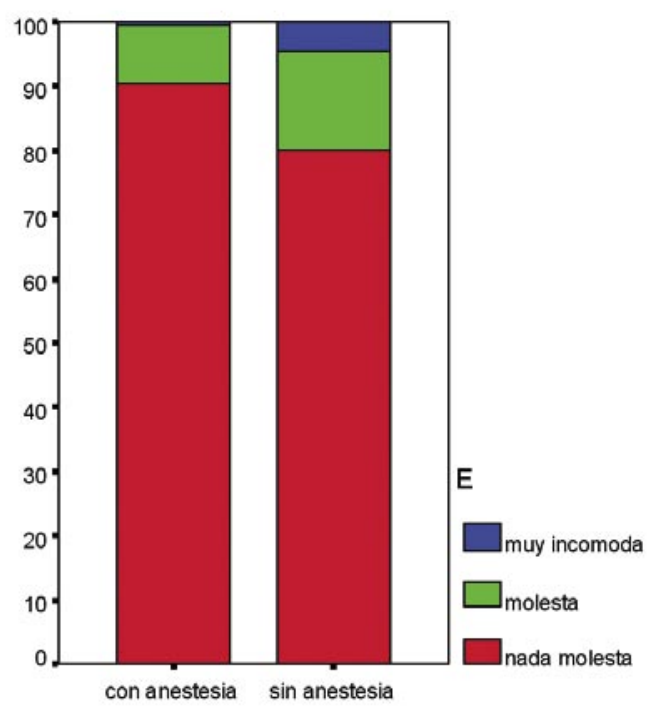

FIGURA 2E. La posición en la camilla le ha resultado

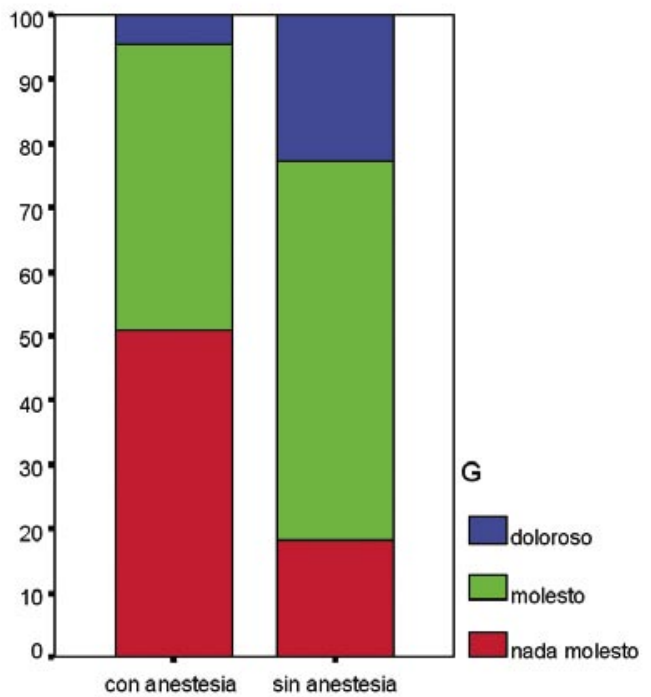

FIGURA 2G. La ecografía y la biopsia le han parecido

Respecto a la rectorragia, cuatro pacientes $(2 \%)$ requirieron ingreso por sangrado importante acompañado de hipotensión y disminución o perdida de conocimiento. En dos de ellos se procedió a la realización de rectoscopia y esclerosis del punto sangrante mediante norepinefrina. En uno de estos pacientes el sangrado apareció una semana después de la BTE; el paciente tomaba antiagregantes (Plavix®) que había reintroducido 72 horas antes. En otros dos se realizó taponamiento rectal. Ninguno precisó de transfusión sanguínea.

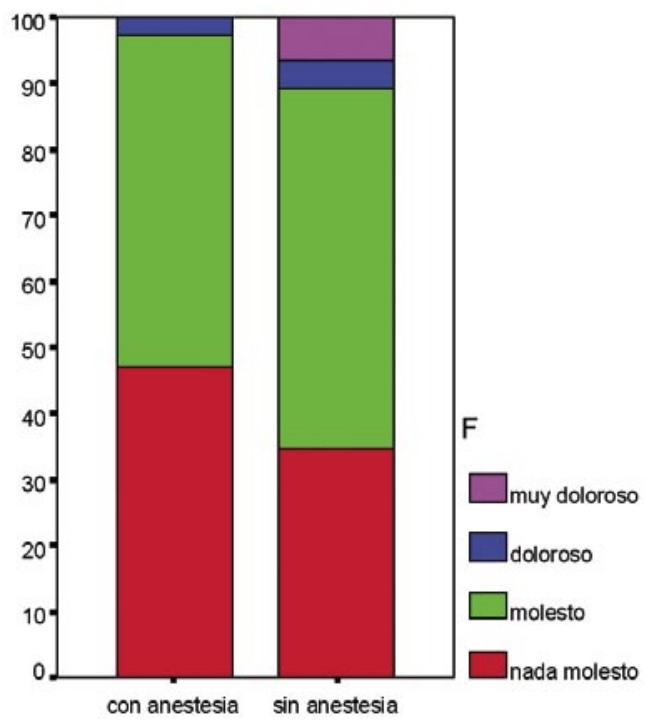

FIGURA 2F. El tacto rectal y el paso del aparato le han parecido

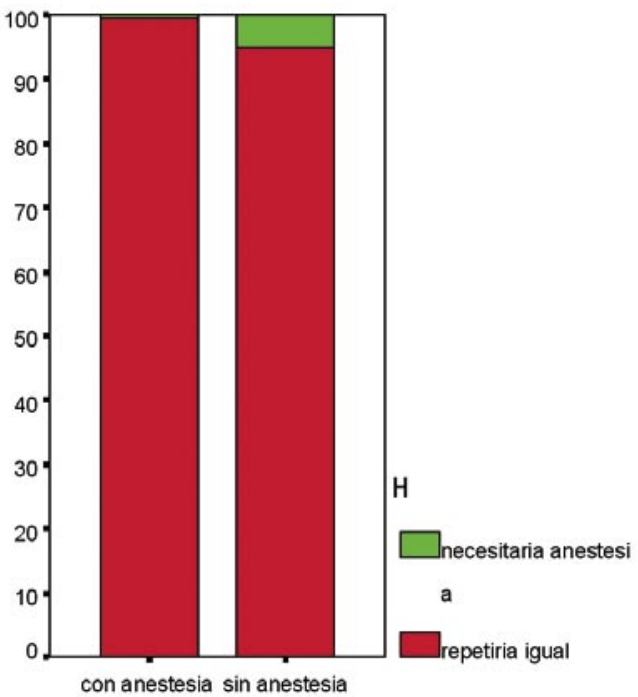

FIGURA 2H. Si tuviera que repetir la biopsia

Presentaron rectorragia moderada-abundante cinco pacientes $(2,57 \%)$. Su manejo se incluye en la tabla 2. En total supone que un 4,7\% (9/194) presentaron una rectorragia moderada o importante, $3 \%$ precisaron asistencia médica y $2 \%$ tratamiento e ingreso mayor de 24 horas.

Únicamente se ha registrado un episodio febril mayor de $38^{\circ}$ en un paciente cuya anatomía patológica reflejó prostatitis granulomatosa necrotizante. Interrogado el paciente presentaba febrícula 
mantenida previa a la BTE y con posterioridad desarrolló una tuberculosis sistémica con afectación vertebral de la que tuvo que ser intervenido. En el momento de contactar con él aún presentaba febrícula mantenida a pesar del tratamiento tuberculostático.

Se recogió un cuadro de inflamación testicular no febril que fue atendido en urgencias y tratado con antibiótico y antiinflamatorios con buena respuesta clínica.

La Tabla II recoge la incidencia de estas complicaciones mayores y el resto de efectos adversos/ complicaciones se recogen en la Tabla III.

El efecto secundario más frecuente es la hematuria que sólo en una ocasión se acompañó de pequeños coágulos.

No precisó atención específica en ningún caso aunque uno de los pacientes acudió al Servicio de Urgencias al aparecer unas pequeñas perdidas sanguíneas por uretra unos días después de la BTE.
La hemospermia aparece también en casi la mitad de los pacientes.

No se ha presentado ningún caso de retención urinaria.

\section{DISCUSIÓN}

Aunque la biopsia transrectal ecodirigida se ha considerado desde el inicio como una prueba bien tolerada y de escasas complicaciones, queda claro que es una exploración cuando menos molesta y que en algunos pacientes llega a ser dolorosa o imposible de realizar. Existe cierta controversia sobre la influencia del número de muestras y la tolerancia. Leibovici y cols (4) encuentran relación entre el número de cilindros y el dolor referido mientras que Naughton y cols (5) no refieren cambios en la apreciación del dolor tomando 6 o 12 muestras. Rodríguez-Patrón y cols (3) en biopsia sextante refieren que la tolerancia empeora en el $47 \%$ de los pacientes a medida que se obtienen más muestras. En cualquier caso en

TABLA II. COMPLICACIONES MAYORES.

\begin{tabular}{|l|c|c|c|c|}
\hline Rectorragia & leve & Moderada & Importante & \\
& $24(12,4 \%)$ & $4(2,1 \%)$ & $5(2,6 \%)$ & \\
\hline Tratamiento & observación & taponamiento & Rectoscopia /esclerosis & Atención urgencias o ingreso \\
& $1(0,5 \%)$ & $2(1 \%)$ & $2(1 \%)$ & $6(3 \%)$ \\
\hline Fiebre/ITU & Fiebre* & orquitis & & \\
& $1(0,5 \%)$ & $1(0,5 \%)$ & & \\
\hline
\end{tabular}

Datos recogidos sobre 194 pacientes contactados.

* Paciente con tuberculosis sistémica.

TABLA III. EFECTOS ADVERSOS Y COMPLICACIONES MENORES DE LA BTE (SOBRE 194 PACIENTES CONTACTADOS).

\begin{tabular}{|llll|}
\hline HEMATURIA & NADA & LEVE & COAGULOS \\
& $67(34,5 \%)$ & $126(64,9 \%)$ & $1(0,5 \%)$ \\
DIFICULTAD MICCIONAL & LEVE & DIFICULTAD EVACUACION & LEVE \\
& $1(0,5 \%)$ & & $4(2,1 \%)$ \\
ESCOZOR MICCIONAL & LEVE & MODERADO & \\
HEMOSPERMIA & $5(2,6 \%)$ & $3(1,5 \%)$ & NS/NC \\
& NO & SI & $72(371)$ \\
DIARREA & $38(19,6 \%)$ & $84(43,29 \%)$ & $1(0,5 \%)$ \\
MOLESTIAS INESPECÍFICAS & $1(0,5 \%)$ & DOLOR PERINEAL & $1(0,5 \%)$ \\
\hline
\end{tabular}


los últimos años, en paralelo a la creciente implantación de biopsias ampliadas, se ha desarrollado un mayor interés por la posibilidad de añadir algún tipo de anestesia que, sin que convierta la prueba en algo engorroso y mucho más complejo, permita su realización de forma más confortable y llegado el caso obtener mayor número de cilindros. Nash y cols (7) realizan la primera descripción del bloqueo de haces neurovasculares para la BTE con lidocaina al $1 \%$ en el ángulo formado entre las vesículas seminales y la base prostática, modelo en el que se basó la aplicación de anestesia en esta serie. Posteriormente se han descrito otras técnicas como la administración única en el ápex prostático (7), la punción combinada de ápex, haces y bordes laterales de la próstata (8) o en diafragma urogenital, bandeletas neurovasculares y espacio recto-prostático (9).

El porcentaje de pacientes en los que no pudo completarse la biopsia es un dato objetivo que resultó mayor de forma significativa en aquellos que no se empleó anestesia. Hasta donde se ha revisado la literatura es difícil encontrar estudios que refieran en cuantos pacientes no se puede realizar o completar la biopsia por mala tolerancia, siendo claro que al menos un porcentaje de ellos presentarán anomalías anorrectales que impidan el paso del transductor o lo hagan excesivamente doloroso.

En un trabajo previo de nuestro grupo (3) sobre tolerancia y complicaciones en biopsia sextante no se pudo completar la toma de muestras en el 1,2\% de pacientes; de los cuatro pacientes en tres no se llegó a obtener ninguna muestra debido a que no toleraron el paso del transductor o su permanencia en el recto. Este porcentaje fue mayor en el "grupo CA" y "grupo $\mathrm{SA}^{\prime \prime}(2,3 \%$ y $16 \%$ respectivamente) a pesar de que en parte se empleara bloqueo neurovascular, que no debe influir en el dolor por la dilatación anal, de ahí que el número de pacientes que no toleren la primera parte de la BTE no dependa tanto del empleo de anestesia como de la incidencia en el grupo de fisura anal, estenosis esfinterianas, hipertonía u otros problemas a este nivel la pesar de que en este estudio no se ha encontrado asociación con las mismas). Posiblemente el grado de ansiedad previa también influya; de los 4 pacientes a los que puntuamos con el grado mayor, en tres no se completó la prueba, si bien en el resto de niveles no hay mayor incidencia de pruebas incompletas.

Tampoco se ha evidenciado diferencia con la ansiedad referida por los pacientes a diferencia de Leibovici y cols (4) o Peyromaure y cols (10), que relacionan el dolor con ésta además de con el número de muestras obtenidas. Masood y cols (11) comparando la tolerancia de la prueba empleando oxido nitroso refieren que en el grupo de control no se pudo completar la prueba en el $4,4 \%$ de pacientes. El empleo de gel de lidocaina al $2 \%$ intrarrectal podría ser de cierta utilidad en estos pacientes ya que diversos autores encuentran que es eficaz para disminuir el dolor de la biopsia y Stirling y cols (12) en concreto refieren que el dolor de la introducción del transductor es menor con el empleo previo de estos geles. En nuestra experiencia lo hemos utilizado con éxito en algún paciente con hipertonía anal o con fisura anal que no habían tolerado la introducción previamente, aunque en aquellos con estenosis establecidas del conducto anal probablemente no sea efectivo.

Al comparar la tolerancia de ambos grupos como era de esperar se aprecian diferencias significativas en la apreciación del dolor durante la realización de la ecografía y biopsia y en la apreciación global, pero llama la atención que también resultó más dolorosa la introducción del transductor y la incomodidad de la posición en la camilla lo que sólo puede explicarse porque la influencia de la experiencia posterior fuera mejor o peor.

También mayor porcentaje de pacientes de los realizados sin anestesia consideraron necesario el empleo de algún tipo de anestesia $15 \%$ frente al $0,69 \%)$.

Peyromaure y cols. (10) en pacientes a los que se tomaron 10 muestras sin anestesia refieren que para el $47,6 \%$ resultó dolorosa. Para Taverna y cols (7) son un $35 \%$ obteniendo 12 muestras. Ambos datos contrastan con un 10,8\% de pacientes de esta serie que realizados sin anestesia consideraron la prueba como dolorosa o muy dolorosa.

Coincide con Taverna y cols (7) en que los pacientes con bloqueo de haces neurovasculares refirieron escaso dolor en más del 93\%.

Sólo el 1,91\% de pacientes del "grupo CA" consideraron la BTE como dolorosa o muy dolorosa. En un estudio previo de nuestro grupo sobre tolerancia en biopsia sextante sin anestesia (3) este porcentaje resultó del $6,8 \%$ y ha sido del 10,87\% en el "grupo $\mathrm{SA}^{\prime}$. Se aprecia que efectivamente la tolerancia a 10 muestras con anestesia es mejor que la sextante sin anestesia, aunque para un pequeño porcentaje de pacientes, y que sin el uso de lidocaina el número de cilindros obtenidos influiría en una peor tolerancia.

El porcentaje de pacientes del "grupo SA" que considerarían necesario el empleo de anestesia es similar a lo descrito por Irani y cols (13) y Collins y cols (14), aunque entre los pacientes a los que se administró lidocaina fue mínimo. Sólo el 2,9\% de los 
pacientes sometidos a biopsia sextante sin anestesia consideró necesario el empleo de algún tipo de anestesia (3).

No se encontró relación entre la tolerancia y los grupos de edad lo que debe estar influido por el uso del bloqueo de haces, ya que otros autores han relacionado una menor edad con peor tolerancia $(3$, $15,16)$.

La media de puntuación en la escala visual analógica fue significativamente diferente según se utilizase anestesia o no $(2,46 \pm 1,67$ vs $4,5 \pm 2,11)$ lo que coincide con la mayoría de los autores que han empleado esta técnica independientemente del lugar de infiltración $(4,6,12,17,18,19)$. Únicamente Wu y cols (20) no encuentran beneficio en el uso de lidocaina inyectada frente a placebo. Otros autores a pesar de demostrar eficacia son más partidarios de otras técnicas de anestesia local como el gel de lidocaina al $2 \%$ intrarrectal que añade la mayor facilidad de aplicación y menor manipulación invasiva $(21,22)$. Sin embargo diferentes trabajos comparativos han encontrado mejor control del dolor de la BTE con el bloqueo de haces $(9,12)$ o no han conseguido encontrar diferencias del gel frente a placebo (23).

También se ha descrito el empleo de óxido nitroso con resultados satisfactorios $(11,24)$ pero esto obliga a disponer de toma de gases y cierta monitorización del paciente que complica la realización de la prueba.

Los únicos efectos secundarios asociados claramente a la inyección de la lidocaina fueron la aparición de un leve mareo, de alteraciones auditivas y de "sabor metálico" o parestesias linguales en 6 pacientes. Estos efectos se relacionan con el paso intravascular del fármaco y aunque siempre se aspira para comprobar que la aguja no se encuentra en el interior de un vaso, debe haberse producido un mínimo paso al torrente sanguíneo. En todos lo casos se resolvieron antes de terminar la prueba y posiblemente podrían evitarse con la inyección de menos volumen y más lentamente.

En general la mayoría de los autores no encuentran efectos adversos asociados a la administración del anestésico local. Öbek y cols (17) comparan las complicaciones de un grupo al que se realizó bloqueo de haces neurovasculares frente a otro sin anestesia y aunque no demuestra diferencias significativas, presentaron fiebre mayor de $38^{\circ}$ o cultivo de orina positivo un mayor porcentaje de pacientes a los que se administró lidocaina 10 y $22 \%$ vs 2 y $6 \%$ respectivamente), además de 2 ingresos por fiebre entre éstos.
Como cualquier prueba invasiva la BTE no está exenta de efectos adversos y complicaciones. En 1971 Davison y col (25) referían un $27,4 \%$ de fiebre, un $44 \%$ y un $3,5 \%$ de cultivos urinarios y hemocultivos positivos respectivamente y un fallecimiento por sepsis en 113 pacientes a los que se realizó biopsia transrectal con aguja de Vim-Silverman. Entonces la profilaxis se realizaba con tetraciclinas, sulfamidas, furantoina o ampicilina que no resultan las más adecuadas, pero a pesar de ello la probabilidad de fiebre era doble en pacientes sin profilaxis. Incluso con PAAF en lugar de biopsia Esposti y cols (26) encuentran un $7,1 \%$ de infección clínica con un fallecimiento, si bien el autor lo asocia a pacientes con artritis reumatoide $u$ otras poliartritis como factor de riesgo. El sangrado por vía rectal o la hematuria importante también podían constituir un problema importante $(27,28)$. Afortunadamente la introducción de la BTE mediante agujas precargadas en dispositivos de disparo automático trajo consigo además de una mejor tolerancia una disminución de las complicaciones ayudado por el avance en la antibioterapia.

Respecto a las complicaciones encontradas en esta serie, la más inmediata es la reacción vagal, más frecuente en el grupo realizado sin anestesia presentándose de forma leve o moderada en el $28 \%$, cifra superior al $11,2 \%$ encontrado por nuestro grupo en la biopsia sextante (3), un dato más que apoya la peor tolerancia a medida que se amplia el número de muestras en ausencia de anestesia. Distintos autores refieren cifras entre el 1 y el $5,3 \%(15,16,29)$. Habitualmente ceden de forma espontánea o con atropina parenteral.

La rectorragia es la complicación más preocupante para el paciente. Fue moderada o importante en el $4,7 \%$, precisando en el $3 \%$ de los pacientes ingreso al menos de 24 horas y en el $2 \%$ tratamiento específico. Esto contrasta con el $1,3 \%$ de sangrados moderados-importantes en una publicación anterior que recogía las complicaciones en biopsia sextante (3), lo que de alguna forma indicaría la relación entre el número de muestras y la posibilidad de rectorragia, aspecto que recogen Rodríguez y Terris (15) y que parece apoyado por la lógica. Otros autores no han encontrado sin embargo diferencias en las complicaciones según el número de muestras tomadas (5).

La incidencia de rectorragia moderada o importante en la literatura es variable, oscilando entre el 0 y el $9,1 \%(5,10,15,16,30,31,32)$ necesitando ingreso en el $0-1 \%(3,15,29)$.

Sólo se registró un episodio de fiebre mayor de $38^{\circ}$ (en el paciente referido que desarrolló una 
tuberculosis vertebral) y un cuadro de inflamación testicular. A pesar de que en esta serie la incidencia de procesos infecciosos incluyendo el citado es baja, estas complicaciones son las que pueden revestir mayor gravedad con posibilidad de sepsis en el 0,1-0,2\% de pacientes $(29,30)$ y otros cuadros severos incluida la posibilidad de fallecimiento (33-35).

La Tabla IV recoge la incidencia de complicaciones y efectos adversos en la literatura.

Al margen de los referidos anteriormente, el resto de eventos pueden ser considerados como efectos colaterales más que como complicaciones. La hematuria como efecto secundario más frecuente no suele revestir gravedad pero al igual que la hemospermia es una fuente frecuente de consulta sobre todo cuando se prolonga más allá de los primeros días, a pesar de ser explicado al finalizar la prueba y figurar en la hoja de información. También es excepcional la aparición de retención aguda de orina (RAO) dado que las muestras se obtienen lejos de la uretra. Esto cambia en las biopsias de saturación donde el mayor número de punciones y la cercanía de algunos a la uretra pueden favorecer la aparición de edema o hematoma de la glándula ocasionando más frecuentemente RAO - hematuria importante. Fleshner y cols (39) refieren un $11 \%$ de RAO tras biopsias con 32 a 38 cilindros,
Borboroglu y cols (40) con una media de 22 punciones un $10,5 \%$ de retenciones y Stewart y cols (41) tomando de 14 a 45 muestras un $4,5 \%$ además de un $5 \%$ de hematurias que precisaron ingreso. En caso de obtener un alto número de muestras parece que la vía perineal presenta menor número de estas complicaciones $(42,43)$.

Sería también importante comprobar si el número de complicaciones varía en sucesivas biopsias. En este sentido el estudio de Diavan y cols (30) es muy clarificador observando que, en cuatro biopsias consecutivas, mientras la incidencia de reacciones vagales desciende y mejora la tolerancia, se mantiene la posibilidad de fiebre entre un 2,3 y $3,1 \%$, la de infección urinaria entre el 9,9 y el 12,2\%, la de sepsis entre el 0 y el $0,1 \%$ y la de hematuria severa entre el 0,5 y el $0,8 \%$. Sin embargo, el riesgo de sangrado rectal que necesita observación o taponamiento aumenta aunque de forma no significativa con un $2,1 \%, 2,4 \%, 5,2 \%$ y $9,1 \%$ en la primera, segunda, tercera y cuarta biopsias respectivamente o la disuria de un $7,2 \%, 6,8 \%, 11,2 \%$ y $12,2 \%$ en los mismos casos. Esto reflejaría que un paciente que se someta a tres biopsias por ejemplo asumiría un riesgo total de tener rectorragia moderada o importante del $9,7 \%$ y del $18,8 \%$ si llegara a la cuarta, o una posibilidad del $8,3 \%$ y del $10,9 \%$ respectivamente de fiebre.

\section{TABLA IV. COMPLICACIONES Y EFECTOS ADVERSOS DE LA BIOPSIA EN LA LITERATURA.}

\begin{tabular}{|c|c|c|c|c|c|c|c|c|c|c|c|c|c|}
\hline porcentajes & $\begin{array}{l}\overline{\overline{0}} \\
\stackrel{0}{D} \\
\stackrel{0}{0} \\
0\end{array}$ & 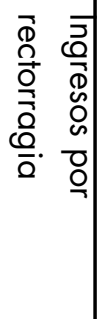 & 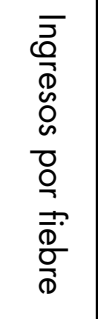 & 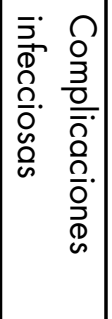 & $\begin{array}{l}\overline{7} \\
\overline{\bar{D}} \\
\frac{0}{\mathbb{D}} \\
\hat{\Lambda} \\
\hat{\omega} \\
0 \\
10\end{array}$ & $\begin{array}{l}\bar{T} \\
\overline{\bar{D}} \\
\frac{0}{\vec{D}} \\
\mathbb{D} \\
\omega \\
0 \\
0 \\
10\end{array}$ & $\begin{array}{l}\frac{\mathscr{D}}{0} \\
\frac{\omega}{\omega}\end{array}$ & 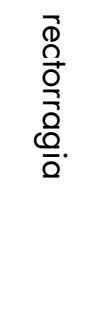 & 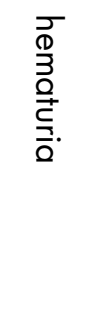 & $\begin{array}{l}\bar{\Phi} \\
\frac{\Phi}{3} \\
0 \\
\frac{0}{0} \\
\mathbb{1} \\
3 \\
\bar{\Omega} .\end{array}$ & 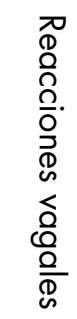 & 㞧 & $\begin{array}{l}\frac{0}{9 .} \\
\stackrel{5}{0} .\end{array}$ \\
\hline R. Patrón (3) & 1 & 1 & 0 & 1,8 & 1,1 & 0,7 & 0 & 22,3 & 51,1 & 21,3 & 2,4 & 0,3 & 2,8 \\
\hline Collins (14) & & & & & & 4 & & 37 & 58 & 29 & & 0 & 7 \\
\hline Rodríguez (15) & & 0 & & & & 2,5 & & 8,2 & 47 & 9 & 5,3 & 1,5 & 9 \\
\hline Rietbergen (29) & 0,4 & 0 & 0,4 & & 4 & 4,2 & 0,2 & 1,7 & 23 & 45 & 1 & 0,45 & \\
\hline Diavan (30) & & & & 10,9 & & 2,9 & 0,1 & 2,1 & 62 & 9,8 & 2,8 & 1,8 & 7,2 \\
\hline Enlund (36) & & 0,2 & 0,75 & & & 2,9 & & 21,7 & 49,6 & & & 0,2 & 13,7 \\
\hline Lujan (37) & & & 1,9 & 11,5 & & & & 25,4 & 47,8 & 31,3 & & 3 & \\
\hline Clements (38) & & & & & & 2,5 & & 7,5 & 20 & 11 & & 1,2 & \\
\hline
\end{tabular}




\section{CONCLUSIONES}

A la vista de lo anteriormente expuesto, es difícil aceptar por lógica que el aumento de muestras aumente el diagnóstico pero no las complicaciones; el limitado número de pacientes reunido en la mayoría de las publicaciones impide establecer con seguridad el porcentaje de complicaciones de la técnica, ya de por sí escasos. En nuestra experiencia se produce un incremento de incidentes sobre todo hemorrágicos con la biopsia ampliada.

El método investigado de anestesia prostática bloqueando los haces neurovasculares con lidocaina se ha mostrado muy eficaz, simple y bien tolerado. La valoración del dolor mediante escala analógica visual lo demuestra, pero para la realización de una biopsia sextante no queda tan siendo probablemente más importante poderla realizar en 3-4 minutos.

Nuestra actitud actual, dado que el efecto anestésico es casi inmediato, es la aplicación de lidocaina sólo a aquellos pacientes que refieren dolor con la primera punción o con mala tolerancia a biopsias previas.

\section{BIBLIOGRAFÍA y LECTURAS RECOMENDADAS ( ${ }^{*}$ lectura de interés $y^{* *}$ lectura fundamental)}

1. HENDRY, W.F.; WILLIAMS, J.P.: "Transrectal prostatic biopsy”. Br. Med. J., 4: 595, 1971.

2. BERTELSEN, S.: "Transrectal needle biopsy of the prostate". Acta Chir. Scand. Suppl., 357: 226, 1966.

*3. RODRÍGUEZ-PATRÓN, R.; MAYAYO, T.; LENNIE, A. y cols.: "Complicaciones de la biopsia transrectal ecodirigida prostática y tolerancia según el paciente y el realizador. Estudio de 305 pacientes". Arch. Esp. Urol. 55: 509, 2002.

*4. LEIBOVICI, D.; ZISMAN, A.; SIEGEL, Y.I. y cols.: "Local anesthesia for prostate biopsy by periprostatic lidocaine injection: a double-blind placebo controlled study". J. Urol., 167: 563, 2002.

*5. NAUGHTON, C.K.; ORNSTEIN, D.K.; SMITH, D.S. y cols.: "Pain and morbidity of transrectal ultrasound guided prostate biopsy: a prospective randomized trial of 6 versus 12 cores". J. Urol., 163: 168, 2000.

**6. NASH, P.A.; BRUCE, J.E.; INDUDHARA, R. y cols.: "Transrectal ultrasound guided prostatic nerve blockade eases systematic needle biopsy of the prostate". J. Urol., 155: 607, 1996.
7. 7. TAVERNA, G.; MAFFEZZINI, M.; BENETTI, A. y cols.: "A single injection of lidocaine as local anesthesia for ultrasound guided needle biopsy of the prostate". J. Urol., 167: 222, 2002.

8. SOLOWAY, M.S.; OBEK, C.: "Periprostatic local anesthesia before ultrasound guided prostate biopsy". J. Urol., 163: 172, 2000.

9. MATLAGA, B.R.; LOVATO, J.F.; HALL, M.C.: "Randomized prospective trial of a novel local anesthetic technique for extensive prostate biopsy". Urology, 61: 972, 2003.

10. PEYROMAURE, M.; RAVERY, V.; MESSAS, A. y cols.: "Pain and morbidity of an extensive prostate 10-biopsy protocol: a prospective study in 289 patients". J. Urol., 167: 218, 2002.*

11. MASOOD, J.; SHAH, N.; LANE, T. y cols.: "Nitrous oxide (entonox) inhalation and tolerance of transrectal ultrasound guided prostate biopsy: a double-blind randomized controlled study". J. Urol., 168: 116, 2002.

12. STIRLING, B.N.; SHOCKLEY, K.F.; CAROTHERS, G.G. y cols.: "Comparison of local anesthesia techniques during transrectal ultrasoundguided biopsies". Urology, 60: 89, 2002.

*13. IRANI, J.; FOURNIER, F.; BON, D. y cols.: "Patient tolerance of transrectal ultrasound-guided biopsy of the prostate". Br. J. Urol., 79: 608, 1997.

*14. COLLINS, G.N.; LLOYD, S.N.; McELVIE, G.B.: "Multiple transrectal ultrasound-guided prostatic biopsies true morbidity and patient acceptance". Br. J. Urol., 71: 463, 1993.

**15. RODRIGUEZ, L.V.; TERRIS, M.K.: "Risks and complications of transrectal ultrasound guided prostate needle biopsy: a prospective study and review of the literature". J. Urol., 160: 2115, 1998.

**16. DJAVAN, B.; WALDERT, M.; ZLOTTA, A. y cols.: "Safety and morbidity of first and repeat transrectal ultrasound guided prostate needle biopsies: results of a prospective European prostate cancer detection study”. J. Urol., 166: 856, 2001.

17. OBEK, C.; ONAL, B.; OZKAN, B. y cols.: "Is periprostatic local anesthesia for transrectal ultrasound guided prostate biopsy associated with increased infectious or hemorrhagic complications? A prospective randomized trial". J. Urol., 168: 558, 2002.

*18. BERGER, A.P.; FRAUSCHER, F.; HALPEERN, E.J. y cols.: "Periprostatic administration of local anesthesia during transrectal ultrasound-guided biopsy of the prostate: a randomized, doubleblind, placebo-controlled study". Urology, 61: $585,2003$.

19. KAVER, I.; MABJEESH, N.J.; MATZKIN, H.: "Randomized prospective study of periprostatic local anesthesia during transrectal ultrasound-gui- 
ded prostate biopsy". Urology, 59: 405, 2002.

20. WU, C.L.; CARTER, H.B.; NAQIBUDDIN, M. y cols.: "Effect of local anesthetics on patient recovery after transrectal biopsy". Urology, 57: 925, 2001.

21. MALLICK, S.; HUMBERT, M.; BRAUD, F. y cols.: "Local anesthesia before transrectal ultrasound guided prostate biopsy: comparison of 2 methods in a prospective, randomized clinical trial". J. Urol., 171: 730, 2004.

22. Randomized prospective trial of intrarectal lidocaine for pain control during transrectal prostate biopsy: The Emory University experience". J. Urol., 164: 397, 2000.

23. CHANG, S.S.; ALBERTS, G.; WELLS, N. y cols.: "Intrarectal lidocaine during transrectal prostate biopsy: results of a prospective double-blind randomized trial". J. Urol., 166: 2178, 2001.

*24. MANIKANDAN, R.; SRIRANGAM, S.J.; BROWN, S.C. y cols.: "Nitrous oxide vs periprostatic nerve block with $1 \%$ lidocaine during transrectal ultrasound guided biopsy of the prostate: a prospective, randomized, controlled trial". J. Urol., 170: 1881, 2003.

25. DAVISON, P.; MALAMENT, M.: "Urinary contamination as a result of transrectal biopsy of the prostate". J. Urol., 105: 545, 1971.

26. ESPOSTI, P.L.; ELMAN, A.; NORLEN, H.: "Complications of transrectal aspiration biopsy of the prostate". Scand. J. Urol. Nephrol., 9: 208, 1975.

27. SONNENSCHEIN, R.: "The effectiveness of transrectal aspiration cytology in the diagnosis of prostatic cancer". Eur. Urol., 1: 189, 1975.

28. OSTROFF, E.B.; ALMARIO, J.; KRAMER, H.: "Transrectal needle method for biopsy of the prostate: review of 90 cases". Am. Surg., 41: 659, 1975.

**29. RIETBERGEN, J.B.; KRUGER, A.E.; KRANSE, R. y cols.: "Complications of transrectal ultrasound-guided systematic sextant biopsies of the prostate: evaluation of complication rates and risk factors within a population-based screening program". Urology, 49: 875, 1997.

30. DJAVAN, B.; RAVERY, V.; ZLOTTA, A. y cols.: "Prospective evaluation of prostate cancer detected on biopsies 1, 2, 3 and 4: when should we stop?". J. Urol., 166: 1679, 2001.

31. DE LA TAILLE, A. ; ANTIPHON, P.; SALOMON, L. y cols.: "Prospective evaluation of a 21-sample needle biopsy procedure designed to improve the prostate cancer detection rate". Urology, 61: 1181, 2003.
32. EMILIOZZI, P.; CORSETTI, A.; TASSI, B. y cols.: "Best approach for prostate cancer detection: a prospective study on transperineal versus transrectal six-core prostate biopsy". Urology, 61: 961, 2003.

33. 33. RODRÍGUEZ-PATRÓN, R.; NAVAS, E.; QUEREDA, C. y cols.: "Meningitis por E. coli multirresistente tras biopsia transrectal ecodirigida". Actas Urol. Esp., 27: 305, 2003.

34. BORER, A.; GILAD, J.; SIKULER, E. y cols.: "Fatal Clostridium Sordellii ischio-rectal abscess with septicaemia complicating ultrasound guided transrectal prostate biopsy". J. Infect., 38: 128, 1999.

35. DA SILVA, E.; PERREIRO, B.; GARIMADI, S. y cols.: "Peritonitis post-biopsia transrectal de próstata". Arch. Esp. Urol., 52: 167, 1999.

36. ENLUND, A.L.; VARENHORST, E.: "Morbidity of ultrasound-guided transrectal core biopsy of the prostate without prophylactic antibiotic therapy. A prospective study in 415 cases". Br. J. Urol., 79: 777, 1997.

*37. LUJAN, M.; PAEZ, I.; FERNÁNDEZ, I. y cols.: "Efectos adversos de la biopsia prostática transrectal. Un análisis de 303 procedimientos". Actas Urol. Esp., 25: 46, 2001.

38. CLEMENTS, R.; AIDEYAN, O.U.; GRIFFITHS, G.J. y cols.: "Side effects and patient acceptability of transrectal biopsy of the prostate". Clin. Radiology, 47: 125, 1993.

39. FLESHNER, N.; KLOTZ, L.: "Role of "saturation biopsy" in the detection of prostate cancer among difficult diagnostic cases". Urology, 60: 93, 2002.

40. BORBOROGLU, P.G.; COMER, S.W. y cols.: "Extensive repeat transrectal ultrasound guided prostate biopsy in patients with previous benign sextant biopsies". J. Urol., 163: 158, 2000.

41. STEWART, C.A.; LEIBOVICH, B.C.; WEAVER, A.L. y cols.: "Prostate cancer diagnosis using a saturation needle biopsy technique after previous negative sextant biopsies". J. Urol., 166: 86, 2001.

42. EMILIOZZI, P.; SCARPONE, P.; DEPAULA, F. y cols.: "The incidence of prostate cancer in men with prostate specific antigen greater than 4.0 $\mathrm{ng} / \mathrm{ml}$ : a randomized study of 6 versus 12 core transperineal prostate biopsy". J. Urol., 171: 197, 2004.

43. IGEL, T.C.; KNIGHT, M.K.; YOUNG, P.R. y cols.: "Systematic transperineal ultrasound guided template biopsy of the prostate in patients at high risk". J. Urol., 165: 1575, 2001. 